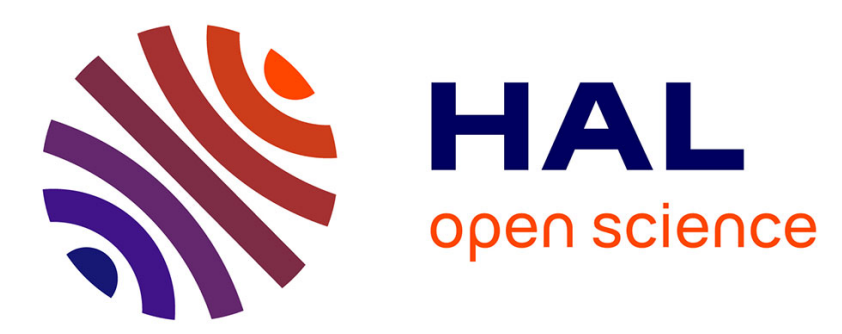

\title{
Analysis and control of Andronov-Hopf oscillators with applications to neuronal populations
}

Elena Panteley, Antonio Loria, Ali El Ati

\section{To cite this version:}

Elena Panteley, Antonio Loria, Ali El Ati. Analysis and control of Andronov-Hopf oscillators with applications to neuronal populations. 54th IEEE Conference on Decision and Control (CDC), Dec 2015, Osaka, Japan. pp.596-591, 10.1109/cdc.2015.7402294 . hal-01262559

\section{HAL Id: hal-01262559}

https://hal-centralesupelec.archives-ouvertes.fr/hal-01262559

Submitted on 5 Mar 2020

HAL is a multi-disciplinary open access archive for the deposit and dissemination of scientific research documents, whether they are published or not. The documents may come from teaching and research institutions in France or abroad, or from public or private research centers.
L'archive ouverte pluridisciplinaire $\mathbf{H A L}$, est destinée au dépôt et à la diffusion de documents scientifiques de niveau recherche, publiés ou non, émanant des établissements d'enseignement et de recherche français ou étrangers, des laboratoires publics ou privés. 


\title{
Analysis and control of Andronov-Hopf oscillators with applications to neuronal populations
}

\author{
Elena Panteley Antonio Loría Ali El Ati
}

\begin{abstract}
One of the main motivations for analysis of networks of nonlinear oscillators lies in the role these play in undertanding synchronization and collective behavior in chemical and biological systems. In medical systems such networks are used for synchronization analysis of neuronal populations and control design for synchrony suppression. In this paper we use tools issued from nonlinear stability theory and graph theory for analysis and control of heterogeneous networked Andronov-Hopf oscillators.
\end{abstract}

\section{INTRODUCTION}

One of the key issues in synchronization analysis of networks of heterogeneous systems pertains to the emergence of new collective phenomena and the manner how individual dynamics, as well as the coupling architecture, affect the arising synchronized dynamics. This is specially the case of large networks of biological oscillators, such as circadian rhythms, heart cells clocks and certain neuronal activity [18]. For instance, the cause-effect of neuronal synchronization and certain brain disorders, such as Parkinson's diseases, is wellrecognized -see [2]. To model and analyze these phenomena, the Andronov-Hopf oscillator is often used, individually or coupled in a network -[19], [6], [8].

From a theoretical viewpoint, issues related to the dependence of the synchronization on the coupling strength among the oscillators, and to the stability properties of the synchronous dynamics, have attracted steadily increasing attention during the last few decades. In the case of heterogeneous networks, the coupled Andronov-Hopf oscillators are only frequency synchronized that is, the amplitudes of their oscillations do not coincide. Different tools, such as Dula's theorem and Lyapunov exponents, have been used in the literature to study stability properties of the limit cycle for a single Andronov-Hopf oscillator and for networks of such oscillators, see e.g., [17]; in [13] Lyapunov type techniques were used to study stability for a network of identical oscillators. However, in the general case of heterogeneous networks of AndronovHopf oscillations, finding the synchronization frequency is a challenging and, to the best of our knowledge, open problem.

In this paper we give an approximate expression for this frequency, which depends on the parameters of the individual oscillators and on the matrix of the interconnections. Furthermore, we present results on synchronization of AndronovHopf oscillators with different oscillating frequencies, under diffusive coupling. The analysis that we carry out relies on the framework of emergent dynamics, presented in [11] which consists in two main parts: the stability analysis of the average

The first two authors are with the CNRS. Address: LSSCENTRALESUPELEC, 91192 Gif-sur-Yvette, France. E-mail: panteley@1ss.supelec.fr dynamics and that of the synchronization manifold corresponding to the synchronization errors.

The rest of the paper is organized as follows. In Section II we describe the network model under study and give a problem formulation. In Sections III and IV we transform the network model in a new set of coordinates suitable for our analysis purposes. In Section V we present our main statements, followed by Section VI where we present a brief case-study of controlled synchronization that includes some numerical simulations. The paper is wrapped up with concluding remarks.

\section{SYNCHRONISATION OF NETWORKED ANDRONOV-HOPF OSCILLATORS}

We consider a network composed of $N$ heterogeneous diffusively coupled Andronov-Hopf oscillators,

$$
\begin{aligned}
\dot{z}_{i} & =f\left(z_{i}, \mu_{i}\right)+u_{i}, \quad i \in \mathcal{I}:=\{1, \ldots, N\} \\
f\left(z_{i}, \mu_{i}\right) & :=-\left|z_{i}\right|^{2} z_{i}+\mu_{i} z_{i}
\end{aligned}
$$

where $z_{i}, u_{i} \in \mathbb{C}$ are, respectively, the state and the input of $i$ th oscillator, $\mu_{i}=\mu_{\mathrm{R} i}+\mathrm{i} \mu_{\mathrm{I} i} \in \mathbb{C}$ is a complex parameter which defines the asymptotic behaviour of the $i$ th oscillator. Heterogeneity of the network is due to the fact that the parameters $\mu_{i} \in \mathbb{C}$ are different for each oscillator.

We assume that the oscillators are interconnected via diffusive coupling, i.e., for the $i$-th oscillator the input is given by

$$
u_{i}=-\gamma\left[d_{i 1}\left(z_{i}-z_{1}\right)+d_{i 2}\left(z_{i}-z_{2}\right) \ldots+d_{i N}\left(z_{i}-z_{N}\right)\right],
$$

where $d_{i j} \geq 0$ for all $i, j \in \mathcal{I}$ and the scalar parameter $\gamma>0$ corresponds to the coupling strength.

In the case of a homogeneous (i.e. $\mu_{i}=\mu_{j}$ for all $i, j \in \mathcal{I}$ ) and symmetric network, all of the systems tend to oscillate at the same frequency and with zero phase differences. This effect, which is called complete network synchronization, may be formulated as a problem of asymptotic stability of the synchronization manifold

$$
\mathcal{S}=\left\{z_{i} \in \mathbb{C}: z_{1}=z_{2}=\ldots=z_{N}\right\}
$$

and it may be broached using analysis tools developed for semi-passive, incrementally passive or incrementally inputoutput stable systems -see [14], [5], [15] among others.

The behaviour of networks of systems with non-identical models is more complex due to the fact that the synchronization manifold $\mathcal{S}$ does not necessarily exist due to the differences among the dynamics of the oscillators. According to the approach introduced in [10] the behaviour of the oscillators interconnected over the network (2), may be completely characterized in terms of two properties: the stability of what 
we call the emergent dynamics and, complementarily, the asymptotic convergence of each unit's motion to that of an averaged system, called mean-field oscillator. The first property is analyzed in terms of input to state stability of the average system dynamics with respect to a decomposable compact invariant set [12]. The second is captured by the stability of the synchronization errors manifold

$$
\mathcal{S}=\left\{\boldsymbol{e} \in \mathbb{C}^{N}: e_{1}=e_{2}=\ldots=e_{N}=0\right\}
$$

where $e_{i}=z_{i}-z_{m}$. However, due to the heterogeneity of the network, perfect synchronization may not be expected in general. Hence, we introduce the following definition of practical stability of a set, which is similar to that of practical stability of an equilibrium point -see [16].

Consider a parameterized system of differential equations

$$
\dot{x}=f(x, \varepsilon),
$$

where $x \in R^{n}$ and the function $f: \mathbb{R}^{n} \rightarrow \mathbb{R}^{n}$ is locally Lipschitz and $\varepsilon>0$ is a scalar parameter. We assume that $\varepsilon \in\left(0, \varepsilon_{0}\right]$. For such a family we introduce the notion of global practical uniform asymptotic stability with respect to closed, not necessarily compact, sets.

Definition 1 For the system (5), we say that the closed set $\mathcal{A} \subset \mathbb{R}^{n}$ is practically uniformly asymptotically stable if there exists a closed set $\mathcal{D}$ such that $\mathcal{A} \subset \mathcal{D} \subset \mathbb{R}^{n}$ and:

(1) the system is forward complete for all $x_{\circ} \in \mathcal{D}$;

(2) for any given $\delta>0$ and $R>0$, there exist $\epsilon^{*} \in\left(0, \epsilon_{\circ}\right]$ and a class $\mathcal{K} \mathcal{L}$ function $\beta_{\delta}$ such that, for all $\epsilon \in\left(0, \epsilon^{*}\right]$ and all $x_{\circ} \in \mathcal{D}$ such that $\left|x_{\circ}\right|_{\mathcal{A}} \leq R$, we have

$$
\left|x\left(t, x_{\circ}, \epsilon\right)\right|_{\mathcal{A}} \leq \delta+\beta_{\delta R}\left(\left|x_{\circ}\right|_{\mathcal{A}}, t\right) .
$$

where $|x|_{\mathcal{A}}:=\inf _{y \in \mathcal{A}}|x-y|$.

\section{NETWORK STRUCTURE}

We assume that the network graph is connected and undirected, moreover, for simplicity we assume that the interconnections weights are real. Then, the corresponding Laplacian matrix $L$ has exactly one eigenvalue (say, $\lambda_{1}$ ) equal to zero, while others are positive, i.e., $0=\lambda_{1}<\lambda_{2} \leq \ldots \leq \lambda_{N}$. Furthermore, we denote the overall network's state by $z \in$ $\mathbb{C}^{N}, \boldsymbol{z}=\left[z_{1}, \ldots, z_{N}\right]^{\top}$. Then, using (1) and the expression for the diffusive coupling, (2), we see that the overall network dynamics can be described by the equation

$$
\dot{z}=F(\boldsymbol{z})-\gamma L \boldsymbol{z},
$$

where the function $F: \mathbb{C}^{N} \rightarrow \mathbb{C}^{N}$ is given by

$$
F(\boldsymbol{z})=\left[f\left(z_{i}, \mu_{i}\right)\right]_{i \in \mathcal{I}} .
$$

In order to analyze the behaviour of the solutions, $\boldsymbol{z}(t)$, of (6) we proceed to rewrite the system dynamics in new coordinates which exhibit the network emergent dynamics. To that end, let us define the diagonal matrices $\mathcal{M}:=$ $\operatorname{diag}\left(\mu_{1}, \ldots, \mu_{N}\right)$ and $C(\boldsymbol{z}):=\operatorname{diag}\left(\left|z_{1}\right|^{2}, \ldots,\left|z_{N}\right|^{2}\right)$, where $\left|z_{i}\right|^{2}:=\bar{z}_{i} z_{i}$ and $\bar{z}_{i}$ corresponds to the complex conjugate of $z_{i}$. Then, we may rewrite the system (6) as

$$
\begin{aligned}
\dot{z} & =A_{\gamma} \boldsymbol{z}-C(\boldsymbol{z}) \boldsymbol{z}, \\
A_{\gamma} & :=\mathcal{M}-\gamma L .
\end{aligned}
$$

Now, if we express the matrix $A_{\gamma}$ as a "perturbed version" of the Laplacian, i.e.,

$$
A_{\gamma}=\gamma(-L+\varepsilon \mathcal{M}), \quad \varepsilon:=\frac{1}{\gamma},
$$

then, for sufficiently large values of $\gamma$, we may use results on perturbation theory for matrices (see, e.g., [4], [9]) to characterize the eigenvalues and eigenvectors of $A_{\gamma}$ in terms of $\varepsilon$ and the eigenvalues and eigenvectors of the Laplacian $L$. In particular, [9, Theorem 2.1], as well as [4], allow to estimate the eigenvalues of $A_{\gamma}$ in terms of those of $L, \mathcal{M}$ and $\varepsilon$. In general, a small perturbation of a matrix $A$ is denoted by

$$
A_{\varepsilon}=A_{0}+\varepsilon A_{1}, \quad \varepsilon \rightarrow 0
$$

so, if we denote by $\lambda_{1}\left(A_{0}\right)$ a simple eigenvalue of $A_{0}$ and by $\lambda_{1 \varepsilon}$ its induced perturbation, then, for sufficiently small $\varepsilon$, we may use the convergent power series representation

$$
\lambda_{\varepsilon}=\lambda_{1}+c_{1} \varepsilon+o(\varepsilon),
$$

in which the coefficient of the first-order term, $c_{1} \varepsilon$, may be characterized as

$$
c_{1}=\frac{w^{\top} A_{1} v}{w^{\top} v}
$$

where $w$ and $v$ are normalized left and right eigenvectors of the unperturbed matrix $A_{0}$ associated to $\lambda_{1}$ hence, $|w|=|v|=1$.

On the other hand, $A_{\gamma} \in \mathbb{C}^{N \times N}$ is complex symmetric, i.e., $A_{\gamma}=A_{\gamma}^{\top}$ and for any symmetric complex matrix $A$ there exists a complex orthogonal matrix $T$, i.e., satisfying $T^{-1}=T^{\top}$, such that $A=T^{\top} M T$ and matrix $M$ has the block-diagonal form $M=\operatorname{diag}\left(M_{1}, M_{2}, M_{3} \ldots\right)$ where each block $M_{k}$ is either scalar, if the eigenvalue is simple, or $M_{k}=\lambda_{k} I+\tilde{M}_{k}$ where $\tilde{M}_{k} \in \mathbb{C}^{q \times q}$, if the eigenvalue has multiplicity $q$, and the eigenvalues of $\tilde{M}_{k}$ equal to zero.

Now, for the system (8a) the matrix $L$ is symmetric and corresponds to a connected graph hence, it is diagonalizable, the left and right eigenvectors of $L$ that correspond to $\lambda_{1}(L)=$ 0 coincide and $w=v=\frac{1}{N} \mathbf{1}$, where $\mathbf{1}:=[1 \cdots 1]^{\top}$.

Thus, by assimilating $A_{\varepsilon}$ in (9) to $(-L+\varepsilon \mathcal{M})$, and consequently $A_{0}$ to $-L$ and $A_{1}$ to $\mathcal{M}$, we see from (11) that

$$
c_{1}=\frac{1}{N} \mathbf{1}^{\top} \mathcal{M} \mathbf{1}=\frac{1}{N} \sum_{i=1}^{N} \mu_{i} .
$$

We deduce that the eigenvalues of $A_{\gamma}$ may be approximated via (10) and

$\lambda_{1}\left(A_{\gamma}\right)=\gamma\left[-\lambda_{1}(L)+c_{1} \varepsilon+o(\varepsilon)\right]=\frac{1}{N} \sum_{i=1}^{N} \mu_{i}+O\left(\frac{1}{\gamma}\right)$.

We conclude that $\lambda_{1}\left(A_{\gamma}\right)$ is bounded as a function of $\gamma$ and it converges to $\frac{1}{N} \sum_{i=1}^{N} \mu_{i}$ as the coupling strength $\gamma \rightarrow \infty$. Moreover, for all $j \in\{2, \ldots, N\}$ we have

$$
\lambda_{j}\left(A_{\gamma}\right)=-\gamma \lambda_{j}(L)+c_{1}+O(\varepsilon)
$$

hence, the eigenvalues of $A_{j}$ are proportional to $\gamma$ and, since $\Re e\left[\lambda_{j}(L)\right]>0$, we have $\Re e\left[\lambda_{j}\left(A_{\gamma}\right)\right] \rightarrow-\infty$ as $\gamma \rightarrow \infty$.

Now, we assume that the following hypothesis holds.

A1 There exists a number $\gamma^{*}>0$ and, for each $\gamma \geq \gamma^{*}$, a diagonal matrix $\Lambda_{\gamma} \in \mathbb{C}^{N \times N}$, whose elements corresponds 
to the eigenvalues of $A_{\gamma}$, and a complex orthogonal matrix $V_{\gamma} \in \mathbb{C}^{N \times N}$, i.e., such that

$$
V_{\gamma}^{\top} V_{\gamma}=I_{N}
$$

and the matrix $A_{\gamma}$ defined in (8b) may be factorized as

$$
A_{\gamma}=V_{\gamma} \Lambda_{\gamma} V_{\gamma}^{-1}
$$

Moreover, there exists $k \leq N$ such that $\Re e\left[\lambda_{k}\right]>$ $\max _{j \in \mathcal{I} \cap j \neq k} \Re e\left[\lambda_{j}\right]$.

Without loss of generality, in what follows we assume that the eigenvalues of $A_{\gamma}$ are ordered decreasingly, that is, $\Re e\left[\lambda_{1}\right]>\Re e\left[\lambda_{2}\right] \geq \ldots \geq \Re e\left[\lambda_{N}\right]$.

We remark that the $i$ th column of the matrix $V_{\gamma}$ corresponds to the right eigenvector, denoted $\vartheta_{\mathbf{r}_{i}}$, associated to the $i$ th eigenvalue of $A_{\gamma}$. Correspondingly, we denote by $\vartheta_{\ell_{i}}$ the $i$ th left eigenvector, which corresponds to the $i$ th row of $V_{\gamma}^{\top}$. Due to the orthogonality of $V_{\gamma}$, expressed by Eq. (12), we have

$$
\left[\vartheta_{\mathbf{r}}\right]^{2}:=\vartheta_{\mathbf{r}}^{\top} \vartheta_{\mathbf{r}}=1
$$

Another important feature of (8) is that it leads to a new representation of the dynamics, which is reminiscent of that of a homogeneous network. To see this, we proceed to decompose the matrix $A_{\gamma}$ as follows. According to Assumption A1, $\Lambda_{\gamma}$ is diagonal hence, let us introduce $\Lambda_{1}, \Lambda_{2}$ such that

$$
\Lambda=\Lambda_{1}+\Lambda_{2}, \quad \Lambda_{1}:=\lambda_{1}\left(A_{\gamma}\right) I_{N},
$$

$\Lambda_{2}:=\operatorname{diag}\left(0, \lambda_{2}\left(A_{\gamma}\right)-\lambda_{1}\left(A_{\gamma}\right), \ldots, \lambda_{N}\left(A_{\gamma}\right)-\lambda_{1}\left(A_{\gamma}\right)\right)$.

Notice that if $\gamma>\gamma^{*}$, where $\gamma^{*}$ satisfies Assumption A1, then $(N-1)$ non-zero eigenvalues of the matrix $\Lambda_{2}$ have negative real parts and, moreover, for all $i \in\{2, \ldots, N\}$ we have $\Re e\left[\lambda_{i}\left(\Lambda_{2}\right)\right] \rightarrow-\infty$ as $\gamma \rightarrow+\infty$.

Using these notations we can rewrite the matrix $A_{\gamma}$ as

$$
A_{\gamma}=V_{\gamma} \Lambda_{1} V_{\gamma}^{\top}+V_{\gamma} \Lambda_{2} V_{\gamma}^{\top}=\lambda_{1}\left(A_{\gamma}\right) I+D,
$$

where $D=V_{\gamma} \Lambda_{2} V_{\gamma}^{\top}$. The interest of the matrix $D$ is that it depends on the systems' parameters $\mu_{i}$ but it inherits the properties of the Laplacian matrix; indeed, in view of the definition of $\Lambda_{2}$ and Assumption A1 we have $D \leq 0$ and, moreover, $N-1$ eigenvalues of this matrix have negative real parts. As a matter of fact, for all $i \in\{2, \ldots, N\}$, we have

$$
\lambda_{i}(D)=\lambda_{i}\left(\Lambda_{2}\right), \Re e\left[\lambda_{i}\left(\Lambda_{2}\right)\right] \rightarrow-\infty \quad \text { as } \quad \gamma \rightarrow+\infty .
$$

The overall conclusion is that the networked system (8a) may be expressed in the following alternative form reminiscent of a network of oscillators with equal parameters $\mu_{i}$ :

$$
\dot{\boldsymbol{z}}=\left[\lambda_{1} I-C(\boldsymbol{z})\right] \boldsymbol{z}+D \boldsymbol{z} .
$$

\section{NETWORK DYNAMICS}

We show that the diagonalizability of $A_{\gamma}$ allows to naturally exhibit the intrinsic emergent dynamics, which is at the core of the networked systems behaviour and, therefore, at the basis of our analysis framework.

Firstly, we represent the system (8) in a coordinates frame whose first coordinate corresponds to a certain "average" of all the units' states. The rest of the coordinates correspond to the synchronization errors. Let

$$
\tilde{\boldsymbol{z}}=V_{\gamma}^{\top} \boldsymbol{z}
$$

and $\widetilde{V}_{\gamma}:=\left[\vartheta_{\mathbf{r}_{2}} \cdots \vartheta_{\mathbf{r}_{\mathrm{N}}}\right]$ then,

$$
\tilde{\boldsymbol{z}}=\left[\begin{array}{c}
\vartheta_{\mathbf{r}_{1}}^{\top} \\
\widetilde{V}_{\gamma}^{\top}
\end{array}\right] \boldsymbol{z} .
$$

From Section III we know that $\lambda_{1}\left(A_{\gamma}\right) \rightarrow \lambda_{1}(L)$ as $\gamma \rightarrow \infty$ and, $\vartheta_{\mathbf{r}_{1}}$, which corresponds to the first right eigenvector of both, $A_{\gamma}$ and $D$, satisfies $\vartheta_{\mathbf{r}_{1}} \rightarrow \mathbf{1}$, as $\gamma \rightarrow \infty$ and $\tilde{\boldsymbol{z}}_{1}$ may be regarded as a weighted average of the units' states $z_{i}$.

Next, let us consider the rest of the coordinates in $\tilde{\boldsymbol{z}}$, i.e., the vector $\tilde{\boldsymbol{z}}_{2}=\widetilde{V}_{\gamma}^{\top} \boldsymbol{z}$. From (12) we have $V_{\gamma}^{\top}=V_{\gamma}^{-1}$, so

$$
\tilde{V}_{\gamma} \widetilde{V}_{\gamma}^{\top}=I_{N}-\vartheta_{\mathbf{r}_{1}} \vartheta_{\mathbf{r}_{1}}^{\top}
$$

and $\tilde{\boldsymbol{z}}_{2}$ equals to zero if and only if $\boldsymbol{z}=\vartheta_{\mathbf{r}_{1}} \tilde{\boldsymbol{z}}_{1}$ or, equivalently, if the synchronization error $\boldsymbol{e}=\boldsymbol{z}-\vartheta_{\mathbf{r}_{1}} \tilde{\boldsymbol{z}}_{1}$ equals to zero.

Thus, the behaviour of the networked systems interconnected via diffusive coupling is naturally and completely captured by the states

$$
\begin{aligned}
z_{m} & :=\vartheta_{\mathbf{r}_{1}}^{\top} \boldsymbol{z} \\
\boldsymbol{e} & :=\boldsymbol{z}-\vartheta_{\mathbf{r}_{1}} z_{m} .
\end{aligned}
$$

By using these coordinates we decompose the analysis of the network behaviour in two distinct parts: the first, pertains to the "average" behaviour of the network and the second, to the synchronization of the units. In what follows, we derive the dynamics equations corresponding to $z_{m}$ and $\boldsymbol{e}$.

To derive the mean-field dynamics we differentiate on both sides of (21a) and use the network dynamics equation (17) to obtain

$$
\begin{aligned}
\dot{z}_{m} & =\vartheta_{\mathbf{r}_{1}}^{\top}\left[\left(\lambda_{1}\left(A_{\gamma}\right) I-C(\boldsymbol{z})\right) \boldsymbol{z}+D \boldsymbol{z}\right] \\
& =\lambda_{1}\left(A_{\gamma}\right) z_{m}-\vartheta_{\mathbf{r}_{1}}^{\top} C(\boldsymbol{z}) \boldsymbol{z}+\vartheta_{\mathbf{r}_{1}}^{\top} D \boldsymbol{z}
\end{aligned}
$$

however, since $\vartheta_{\mathbf{r}_{1}}$ is an eigenvector associated to $\lambda_{1}(D)$, the last term on the right-hand side of (22) equals to zero. We proceed to rewrite the rest of the right-hand side of (22) in terms of $z_{m}$ and $\boldsymbol{e}$. From (21b), we have $C(\boldsymbol{z}) \boldsymbol{z}=C(\boldsymbol{z})\left[\boldsymbol{e}+\vartheta_{\mathbf{r}_{1}} z_{m}\right]$. Denoting $\Gamma(\boldsymbol{z}):=\operatorname{diag}\left(z_{1}, \ldots, z_{N}\right)$, after some tedious but straightforward calculations we obtain

$$
\dot{z}_{m}=\left[\lambda_{1}-\alpha\left|z_{m}\right|^{2}\right] z_{m}+f_{m}\left(z_{m}, \boldsymbol{e}\right)
$$

where

$$
\begin{aligned}
\alpha= & \vartheta_{\mathbf{r}_{1}}^{\top} \Gamma\left(\bar{\vartheta}_{\mathbf{r}_{1}}\right) \Gamma\left(\vartheta_{\mathbf{r}_{1}}\right) \vartheta_{\mathbf{r}_{1}}, \\
f_{m}\left(z_{m}, \boldsymbol{e}\right)= & -\vartheta_{\mathbf{r}_{1}}^{\top}\left[C(\boldsymbol{z})+\Gamma(\boldsymbol{z})^{*} \Gamma\left(\vartheta_{\mathbf{r}_{1}} z_{m}\right)\right] \boldsymbol{e} \\
& -\vartheta_{\mathbf{r}_{1}}^{\top} \Gamma\left(\left[\vartheta_{\mathbf{r}_{11}}^{2} \cdots \vartheta_{\mathbf{r}_{1 \mathrm{~N}}}^{2}\right]^{\top}\right)\left(z_{m}\right)^{2} \overline{\boldsymbol{e}} .
\end{aligned}
$$

We remark that $f_{m} \equiv 0$ if $|\boldsymbol{e}|^{2}=0$ that is, if synchronization is achieved asymptotically the dynamics of the average unit, (23), converges to the emergent dynamics

$$
\dot{z}_{e}=\left[\lambda_{1}-\alpha\left|z_{e}\right|^{2}\right] z_{e} .
$$

Notice, also, that the interconnection gain $\gamma$ does not appear explicitly in the right-hand side of (23). Nonetheless, it easy to 
see from (10) that $\lambda_{1}$ is inversely proportional to $\gamma$. Indeed, roughly speaking, we have $\lambda_{1}(D)=c+O\left(\frac{1}{\gamma}\right)$ where the constant $c$ depends only on the matrix $\mathcal{M}$. The same type of relationship is also valid for the eigenvector $\vartheta_{\mathbf{r}_{1}}$.

Finally, we derive the dynamics equation corresponding to the synchronization error (21b). To that end, let us start by introducing the matrix

$$
P:=\left(I-\vartheta_{\mathbf{r}_{1}} \vartheta_{\mathbf{r}_{1}}^{\top}\right)
$$

hence, we have $e=P \boldsymbol{z}$. Next, differentiating on both sides of the latter and using (17) we obtain the error dynamics of $e$,

$$
\dot{e}=P D \boldsymbol{z}+P\left[\lambda_{1}\left(A_{\gamma}\right) I-C(\boldsymbol{z})\right] \boldsymbol{z} .
$$

Now, since $\vartheta_{\mathbf{r}_{1}}$ is a right eigenvector associated to $\lambda_{1}(D)=0$, it follows that $D P=D=P D$. Therefore, $P D \boldsymbol{z}=P D P \boldsymbol{z}$ and, since $\boldsymbol{e}=P \boldsymbol{z}$, we obtain $P D \boldsymbol{z}=P D \boldsymbol{e}$. It follows from this and (25) that

$$
\dot{\boldsymbol{e}}=\left[D+\lambda_{1}\left(A_{\gamma}\right) I\right] \boldsymbol{e}-P C\left(\boldsymbol{e}+\vartheta_{\mathbf{r}_{1}} z_{m}\right)\left[\boldsymbol{e}+\vartheta_{\mathbf{r}_{1}} z_{m}\right]
$$

Thus, Equations (23) and (26) completely define the dynamics of the networked oscillators and in coordinates meaningful for our purposes of analysis. The next section is devoted to the stability analysis of the solutions of these equations, which we regroup for convenience:

$$
\begin{aligned}
\dot{z}_{m} & =\left[\lambda_{1}-\alpha\left|z_{m}\right|^{2}\right] z_{m}+f_{m}\left(z_{m}, \boldsymbol{e}\right) \\
\dot{\boldsymbol{e}} & =\left[D+\lambda_{1} I\right] \boldsymbol{e}-P C\left(\boldsymbol{e}+\vartheta_{\mathbf{r}_{1}} z_{m}\right)\left[\boldsymbol{e}+\vartheta_{\mathbf{r}_{1}} z_{m}\right]
\end{aligned}
$$

We investigate two different properties. Firstly, we establish a bound on the synchronization errors $e$. Then, we make a statement on stability of the natural attractor of the emergent dynamics (24), which corresponds to the nominal part of (27a).

\section{NETWORKED SYSTEMS' STABILITY}

As a preliminary but fundamental step in the analysis of Equations (27) we formulate conditions that ensure that the trajectories of the networked diffusively-coupled AndronovHopf oscillators, as described by (6) and equivalently by (27), are ultimately bounded. The property may be established for Andronov-Hopf oscillators, for any interconnection gain $\gamma>$ 0 , using simple Lyapunov arguments $-c f$. [13], [7].

Proposition 1 Consider the system (6), (7) and let the graph of the network be undirected and connected. Then, the solutions are globally ultimately bounded and

$$
\begin{aligned}
\left|\boldsymbol{z}\left(t, \boldsymbol{z}_{\circ}\right)\right| & \leq \sqrt{2 \bar{\mu} N}, \quad \forall t \geq T \\
\bar{\mu} & =\max _{i \in \mathcal{I}}\left\{\mu_{R i}, 0\right\} .
\end{aligned}
$$

Based on this and the following statement, which is reminiscent of results found in [3] for stability of an equilibrium, we establish global practical asymptotic stability for the system (27b) with respect to the set $\mathcal{S}$. This implies that for large values of the interconnection gain $\gamma$ the norm of the error $\boldsymbol{e}(t)$ is small and inversely proportional to $\gamma$.

Lemma 1 Consider the system $\dot{\boldsymbol{x}}=f(\boldsymbol{x})$, where $\boldsymbol{x} \in \mathbb{R}^{n}$ and $f: \mathbb{R}^{n} \rightarrow \mathbb{R}^{n}$ is continuous, locally Lipschitz. Assume that the system is forward complete and that there exist a closed set $\mathcal{A} \subset \mathbb{R}^{n}$, a $C^{1}$ function $V: \mathbb{R}^{n} \rightarrow \mathbb{R}_{+}$, functions $\alpha_{1}, \alpha_{2} \in$ $\mathcal{K}_{\infty}, \alpha_{3} \in \mathcal{K}$ and a constant $c>0$ such that

$$
\begin{gathered}
\alpha_{1}\left(|\boldsymbol{x}|_{\mathcal{A}}\right) \leq V(\boldsymbol{x}) \leq \alpha_{2}\left(|\boldsymbol{x}|_{\mathcal{A}}\right) \\
\dot{V} \leq-\alpha_{3}\left(|\boldsymbol{x}|_{\mathcal{A}}\right)+c .
\end{gathered}
$$

Then for any $R, \varepsilon>0$ there exists a $T=T(R, \varepsilon)$ such that for all $t \geq T$ and all $\boldsymbol{x}_{\circ} \in \mathbb{R}^{n}$ such that $\left|\boldsymbol{x}_{\circ}\right|_{\mathcal{A}} \leq R$

$$
\left|\boldsymbol{x}\left(t, \boldsymbol{x}_{\circ}\right)\right|_{\mathcal{A}} \leq r+\varepsilon
$$

where $r=\alpha_{1}^{-1} \circ \alpha_{2} \circ \alpha_{3}^{-1}(c)$.

We are ready to present our main statement of this section; the proof is omitted due to space limitations -see [10].

Theorem 1 Consider the system (6), (7) under Assumption A1. Let $\gamma^{*}$ be such that $\Re e\left[\lambda_{2}\left(A_{\gamma^{*}}\right)\right] \leq 0$. Then, the set $\mathcal{S}$ is uniformly globally practically asymptotically stable for all $\gamma \geq \gamma^{*}$. Moreover, there exist $T^{*}>0, c_{1}, c_{2}>0$, independent of $\gamma$, such that synchronization errors $e(t)$ satisfy

$$
|\boldsymbol{e}(t)|^{2} \leq \frac{c}{\left|\Re e\left[\lambda_{2}\left(A_{\gamma^{*}}\right)\right]\right|} \quad \forall t \geq T^{*} .
$$

Theorem 1 relies mostly upon two properties of the networked system, namely, the negative definiteness of the second smallest eigenvalue of the Laplacian matrix $L$ and uniform boundedness of the trajectories of the network. For a network of the Andronov-Hopf oscillators with coupling gain $\gamma$ it establishes that, for a given arbitrary large ball of initial conditions $B_{R}=\left\{\boldsymbol{z}_{\circ} \in \mathbb{C}^{N}:\left|\boldsymbol{z}_{\circ}\right| \leq R\right\}$ and an arbitrarily small constant $\delta>0$, we can always find constants $\gamma(R, \delta)$ and $T^{*}(R, \delta)$ such that the synchronization errors $\boldsymbol{e}\left(t, \boldsymbol{z}_{\circ}\right)$ satisfy

$$
\left|\boldsymbol{e}\left(t, \boldsymbol{z}_{\circ}\right)\right| \leq \delta \quad \text { for all } t \geq T^{*} .
$$

Finally, to complete our analysis, we consider the behaviour of the solutions $z_{m}(t)$ of (27a). Notice that this equation may be regarded as that of a single Andronov-Hopf oscillator with a perturbation, that is,

$$
\dot{z}_{m}=\left(\lambda_{1}-\alpha\left|z_{m}\right|^{2}\right) z_{m}+u,
$$

with $u=f_{m}\left(z_{m}, \boldsymbol{e}\right)$. Therefore, generally speaking, we may use stability theory for perturbed systems with respect to decomposable sets, as discussed in [1], [12]. Indeed, the origin is an equilibrium point, however, so is the orbit $\left|z_{m}\right|=$ $\sqrt{\lambda_{1 \mathrm{R}} / \alpha_{\mathrm{R}}}$, where $\alpha$ is defined in (24), which is determined by the complex parameters of the systems in the network, $\mu_{i}$. More precisely, the set of equilibria is given by

$$
\mathcal{W}:=\left\{z \in \mathbb{C}:|z|=\sqrt{\frac{\lambda_{1 \mathrm{R}}}{\alpha_{\mathrm{R}}}}\right\} \cup\{z=0\} .
$$

Theorem 2 Consider the network of Andronov-Hopf oscillators defined by Equations (6), (7) and the averaged oscillator of the network defined by (21a), whose dynamics is given by equation (30). Let Assumption A1 be satisfied. Then, the system (30) has the asymptotic gain property and moreover for any $\varepsilon>0$ there exists a gain $\gamma \geq \gamma^{*}$ such that

$$
\limsup _{t \rightarrow+\infty}\left|z_{m}\left(t, z_{\circ}\right)\right|_{\mathcal{W}} \leq \varepsilon .
$$

The proof is omitted due to space limitations -see [10]. 


\section{CASE STUdy: CONTROL DESign USING MEAN FIELD MEASUREMENTS}

Motivated by neuroscience applications and notably by techniques of DBS stimulation, we consider a desynchronization problem for a neuronal network. Similar to [8] we model a population of synchronized neurons as a network of $N$ controlled Andronov-Hopf oscillators, assume that the whole network can be stimulated and that the effect of the external control input $u \in \mathbb{C}$ is the same on all the neurons in the population. Then, analogous to (6), dynamics of the population can be written as

$$
\dot{z}=F(\boldsymbol{z})-\gamma L \boldsymbol{z}+u \mathbf{1},
$$

where $\mathbf{1}=[1, \ldots, 1]^{\top} \in \mathbb{R}^{N}$ and we recall that the function $F: \mathbb{C}^{N} \rightarrow \mathbb{C}^{N}$ is given by

$$
F(\boldsymbol{z})=\left[f\left(z_{i}, \mu_{i}\right)\right]_{i \in \mathcal{I}} .
$$

In this type of applications, measurements of states (outputs) of individual neurons are usually not available, only collective activity of the neurons can be measured and therefore similar to [19], [8] we assume that the only available measurement is mean field of the population defined as

$$
z_{m}=\frac{1}{N} \sum_{i=1}^{N} z_{i}
$$

Notice that in case where all oscillators are identical thus defined mean-field coincides with the definition of the averaged oscillator $z_{m}$ given in (21a).

Following the approach of [19], [8], we pose a desynchronization problem as a problem of (feedback) controller design which ensures that asymptotically $\left|z_{m}(t)\right| \rightarrow 0$ as $t \rightarrow \infty$.

Assuming that the population is composed of identical oscillators and using framework proposed in the previous sections, it is easy to show that a simple feedback proportional to the mean field solves this problem.

Proposition 2 Consider the system (31) with $F(\boldsymbol{z})=$ $\left[f\left(z_{i}, \mu\right]\right.$ and let $\Re(\mu)>0$. Define control input $u$ as

$$
u=-K z_{m}
$$

where control gain $K>0$ is such that $K>\Re(\mu) / N$. Then the sets $\mathcal{S}$ defined by (3) and the set $\mathcal{W}=\left\{\boldsymbol{z}: z_{m}=0\right\}$ are globally asymptotically stable for all $\gamma \geq 0$ and therefore $z_{m}(t) \rightarrow 0$ as $t \rightarrow \infty$.

Sketch of the proof. In the closed loop form, system (31) can be written as

$$
\dot{z}=F(\boldsymbol{z})-\gamma L \boldsymbol{z}-K \mathbf{1 1 ^ { \top }} \boldsymbol{z}=C(\boldsymbol{z})+M \boldsymbol{z}
$$

where $C(\boldsymbol{z})=\operatorname{diag}\left(\left|z_{1}\right|^{2}, \ldots,\left|z_{N}\right|^{2}\right), M:=\mu I-\gamma L-$ $K 11^{\top}$ and we used definition of the mean field $z_{m}$ and (34) to obtain the last term in the first equality.

Since all the oscillators are identical and the average oscillators is defined by (33), the network dynamics can be written in terms of $z_{m}$ and $\boldsymbol{e}=\boldsymbol{z}-z_{m} \mathbf{1}=\left(I-\frac{1}{N} \mathbf{1} \mathbf{1}^{\top}\right) \boldsymbol{z}=P \boldsymbol{z}$ as $\dot{e}=P C(\boldsymbol{z}) \boldsymbol{z}+P M \boldsymbol{z}=P C(\boldsymbol{z}) \boldsymbol{z}+\mu P \boldsymbol{z}-P\left(\gamma L+K \mathbf{1 1} \mathbf{1}^{\top}\right) \boldsymbol{z}$.
Now, since $L$ satisfies $P L=L P$ and $P 11^{\top}=0$ we have

$$
\dot{e}=P C(\boldsymbol{z}) \boldsymbol{z}+\mu \boldsymbol{e}-\gamma L \boldsymbol{e} .
$$

Global asymptotic stability of the set $\mathcal{S}$ may be concluded using Lyapunov function $V_{e}(\boldsymbol{e})=\frac{1}{2}|\boldsymbol{e}|^{2}$. Furthermore, multiplying (35) by $\frac{1}{N} \mathbf{1}^{\top}$ we obtain that the emergent dynamics of the population has the form

$$
\dot{z}_{m}=-\left|z_{m}\right|^{2} z_{m}+(\mu-N K) z_{m} .
$$

Since the coefficient $\Re(\mu)-N K<0$, then using $V_{m}\left(z_{m}\right)=$ $\frac{1}{2}\left|z_{m}\right|^{2}$ we obtain that $z_{m}(t)$ converges to zero.

To illustrate our theoretical findings we have performed a few simulation tests in SIMULINK ${ }^{\mathrm{TM}}$ of MATLAB ${ }^{\mathrm{TM}}$. We consider a network of four diffusively coupled Andronov-Hopf oscillators interconnected according to the Laplacian:

$$
L=\left[\begin{array}{cccc}
-1 & 0 & 1 & 0 \\
0 & -2 & 1 & 1 \\
1 & 1 & -4 & 2 \\
0 & 1 & 2 & -3
\end{array}\right]
$$

The dynamics of the interconnected oscillators is given by

$$
\dot{z}_{j}=\left[-\left|z_{j}\right|^{2}+\mu_{\mathrm{R} j}+\mathrm{j} \omega_{j}\right] z_{j}+\gamma \sum_{i=1}^{N} l_{j i}\left(z_{i}-z_{j}\right)+u_{j}
$$

where the coefficients $l_{j i}$ correspond to the respective elements of $L$ for all $i, j \leq 4, \gamma$ is the interconnection gain and the control input $u_{j}$ is defined as

$$
u_{j}:=-K \sum_{i=1}^{4} z_{i}
$$

We performed four different simulation tests, corresponging respectively, to the four cited figures. In the first two, we use a network of identical oscillators, with parameters

$$
w_{i}=5, \mu_{\mathrm{R} j}=1, \quad \forall i \leq 4 .
$$

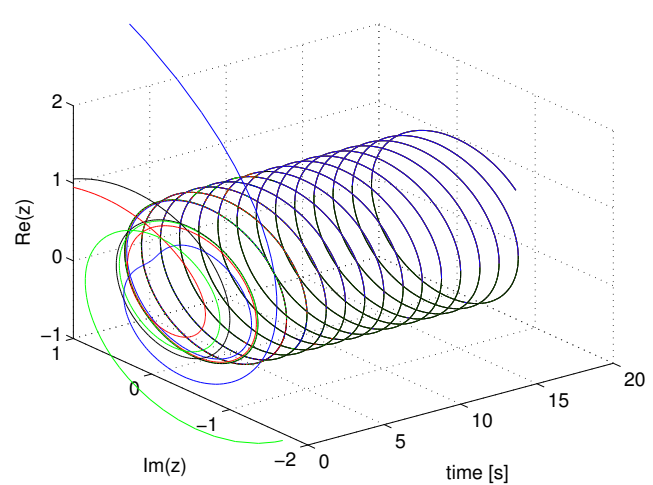

Fig. 1. Mutual synchronization of four identical oscillators without control action $(K=0)$

In Figure 1 we show the phase portrait for the four oscillators perfectly synchronized; in this case, the control action is absent that is, $K=0$. In Figure 2 we show the phase portrait corresponding to the oscillators' behaviours under the action of controller (37) with $K=0.3$. In a second set of simulations, we consider a network of heterogeneous oscillators. We use the numerical parameters: 


$$
\begin{aligned}
& \mu_{\mathrm{R} 1}=-1, \quad \mu_{\mathrm{R} 2}=1, \quad \mu_{\mathrm{R} 3}=1, \quad \mu_{\mathrm{R} 4}=1.5, \\
& w_{1}=-1, \quad w_{2}=1, \quad w_{3}=1, \quad w_{4}=1.5 .
\end{aligned}
$$

As for the first two cases, we firstly performed a numerical test in the absence of control that is, with $K=0$. In Figure 3 we show the evolution of the four phase portraits over time. Finally, in Figure 4 we show the response of the four different interconnected oscillators, each under the action of the controller $u_{j}$ in (37) with control gain $K=0.3$.

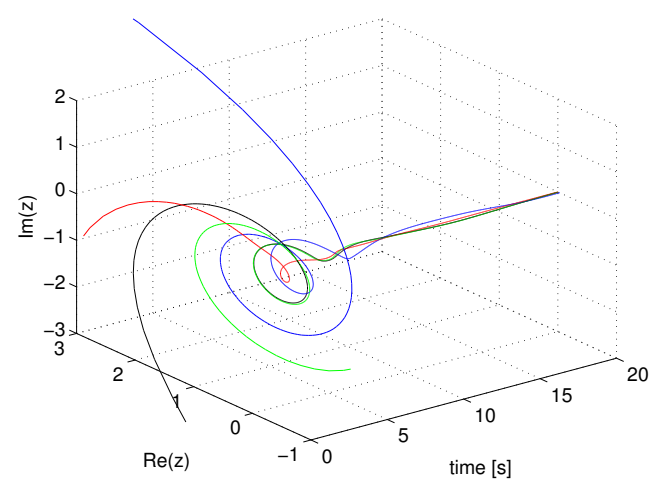

Fig. 2. Phase portrait of mutual synchronization of four identical oscillators with control gain $K=0.3$

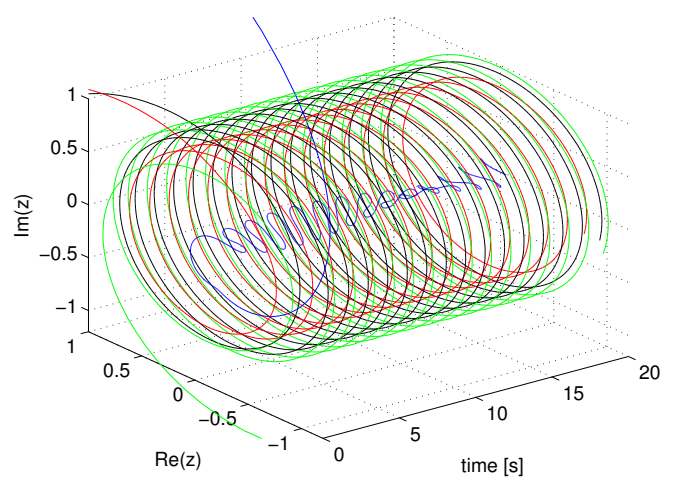

Fig. 3. Phase portrait of mutual synchronization of four different oscillators without control $(K=0)$

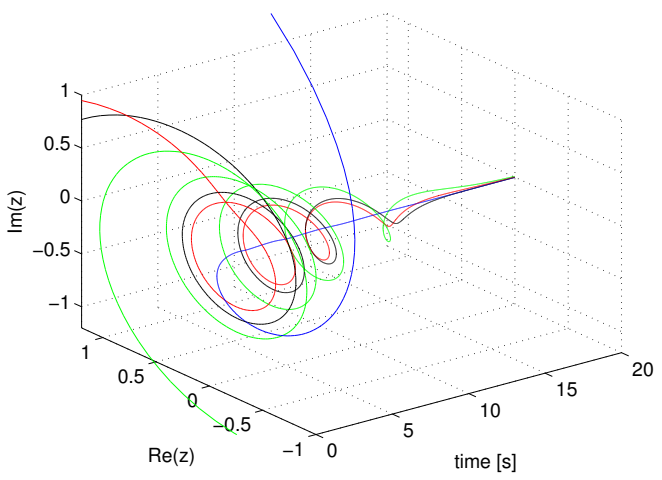

Fig. 4. Phase portrait of mutual synchronization of four different oscillators with control gain $K=0.3$

\section{CONCLUSIONS}

We presented a framework of analysis of synchronization of heterogeneous oscillators described by the Andronov-Hopf equations. The method consists in decoupling the analisis of the collective behaviour, represented by an average oscillator with its own attractor, and the synchronization errors. These are defined, for each agent, relative to the mean-field oscillator. Current research is pursued to develop a control design method based on this analysis framework.

\section{REFERENCES}

[1] D. Angeli and D. Efimov. On input-to-state stability with respect to decomposable invariant sets. In Proc. of the 52nd IEEE Conference on Decision and Control, pages 5897-5902, Florence, Italy, 2013.

[2] H. Cagnan, H. G. Meijer, S. A. Gils, M. Krupa, T. Heida, M. Rudolph, W. J. Wadman, and H. C. Martens. Frequency-selectivity of a thalamocortical relay neuron during Parkinson's disease and deep brain stimulation : a computational study. European Journal of Neuroscience, 30(7):1306-1317, 2009.

[3] M. Corless and G. Leitmann. Continuous state feedback guaranteeing uniform ultimate boundedness for uncertain dynamic systems. IEEE Trans. on Automatic Control, 26(5):1139-1144, 1981.

[4] R. A. Horn and C. R. Johnson. Matrix Analysis. Cambridge Press, 1985.

[5] J. Jouffroy and J. J. Slotine. Methodological remarks on contraction theory. In Proc. of the 43rd IEEE Conference on Decision and Control, volume 3, pages 2537-2543, 2004.

[6] Y. Kuramoto. Chemical oscillations, waves, and turbulence, volume 19. Springer Science \& Business Media, 2012.

[7] P. C. Matthews, R. E. Mirollo, and S. H. Strogatz. Dynamics of a large system of coupled nonlinear oscillators. Physica D: Nonlinear Phenomena, 52:293 - 331, 1991.

[8] G. Montaseri, A. Adhami-Mirhosseini, and M. J. Yazdanpanah. Desynchronization of coupled limit-cycle oscillators through nonlinear output regulation. Systems \& Control Letters, 71:38-43, 2014.

[9] J. Moro, J. Burke, and M. Overton. On the Lidskii-Vishik-Lyusternik perturbation theory for eigenvalues of matrices with arbitrary Jordan structure. SIAM Journal on Matrix Analysis and Applications, 18(4):793-817, 1997.

[10] E. Panteley. A stability-theory perspective to synchronisation of heterogeneous networks. Habilitation à diriger des recherches (Doctor of Sciences Dissertation). Université Paris Sud, Orsay, France, 2015.

[11] E. Panteley, A. Loria, and L. Conteville. On practical synchronization of heterogeneous networks of nonlinear systems: application to chaotic systems. In Proc. IEEE American Control Conference, pages 53595364, Chicago, IL, USA, 2015.

[12] E. Panteley, A. Loria, and A. El-Ati. On the stability and robustness of stuart-landau oscillators. In 1st IFAC Conference on Modelling, Identification and Control of Nonlinear Systems (MICNON), St. Petersburg, Russia, 2015.

[13] Q. C. Pham and J. J. Slotine. Stable concurrent synchronization in dynamic system networks. Neural Networks, 20(1):62-77, 2007.

[14] A. Y. Pogromsky, T. Glad, and H. Nijmeijer. On difffusion driven oscillations in coupled dynamical systems. International Journal of Bifurcation and Chaos in Applied Sciences and Engineering, 9(4):629644, 1999.

[15] L. Scardovi, M. Arcak, and E. D. Sontag. Synchronization of interconnected systems with an input-output approach. Part I: Main results. In Proc. of the 48th IEEE Conference on Decision and Control, pages 609-614, 2009.

[16] A. R. Teel, J. Peuteman, and D. Aeyels. Semi-global practical asymptotic stability and averaging. Systems and Control Letters, 37(5):329-334, 1999.

[17] J. N. Teramae and D. Tanaka. Robustness of the noise-induced phase synchronization in a general class of limit cycle oscillators. Phys. Rev. Lett., 93:204103, 2004.

[18] G. Thut and Minussi. New insight into rhythmic brain activity from tms-eeg studies. Trends Cognit. Sci., 13(1):182-189, 2009.

[19] N. Tukhlina, M. Rosenblum, A. Pikovsky, and J. Kurths. Feedback suppression of neural synchrony by vanishing stimulation. Physical Review E, 75(1), 2007. 